\title{
Echoes of the Brain within the Posterior Cingulate Cortex
}

\author{
Robert Leech, ${ }^{1}$ Rodrigo Braga, ${ }^{1,2}$ and David J. Sharp ${ }^{1}$ \\ ${ }^{1}$ Computational, Cognitive and Clinical Neuroscience Laboratory, Division of Experimental Medicine, Department of Medicine, Hammersmith Hospital \\ Campus, and ${ }^{2}$ MRC Clinical Sciences Centre, Imperial College London, London W12 0NN, United Kingdom
}

There is considerable uncertainty about the function of the posterior cingulate cortex (PCC). The PCC is a major node within the default mode network (DMN) and has high metabolic activity and dense structural connectivity to widespread brain regions, which suggests it has a role as a cortical hub. The region appears to be involved in internally directed thought, for example, memory recollection. However, recent nonhuman primate work provides evidence for a more active role in the control of cognition, through signaling an environmental change and the need to alter behavior. For an organism to flexibly react to a changing environment, information processed in functionally distinct brain networks needs to be integrated by such a cortical hub. If the PCC is involved in this process, its brain activity should show a complex and dynamic pattern that partially reflects activity in other brain networks. Using fMRI in humans and a multivariate analysis, we demonstrate that the PCC shows this type of complex functional architecture, where echoes of multiple other brain networks are seen in separable yet overlapping subregions. For example, a predominantly ventral region shows strong functional connectivity to the rest of the DMN, whereas two subregions within the dorsal PCC show high connectivity to frontoparietal networks involved in cognitive control. PCC subregions showed distinct patterns of activity modulation during the performance of an attentionally demanding task, suggesting that parts of the dorsal PCC interact with frontoparietal networks to regulate the balance between internally and externally directed cognition.

\section{Introduction}

The function of the posterior cingulate cortex (PCC) is uncertain. It is a central node of the default mode network (DMN), a set of brain regions showing strongly correlated neural activity and a reliable deactivation in activity during many cognitive tasks (Raichle et al., 2001). One interpretation of this change in activity is that the region is involved in internally directed thought (Buckner et al., 2008), for example, memory recollection or daydreaming. However, there is converging evidence that the PCC may serve multiple roles, including a more active role in the regulation of cognition (Hampson et al., 2006; Gilbert et al., 2007; Pearson et al., 2011).

The PCC has dense structural connections to many other brain regions, suggesting a role as a cortical hub (Hagmann et al., 2008). It is also one of the most metabolically active brain regions (Raichle et al., 2001), both at rest and during cognitively challenging tasks (Pfefferbaum et al., 2011). Functional connectivity analysis reveals that the PCC and adjacent precuneus are highly heterogeneous (Margulies et al., 2009; Leech et al., 2011). The PCC's functional connectivity dynamically reconfigures in response to changes in task demands, suggesting a role for the dorsal PCC in cognitive control (Leech et al., 2011). Recent elec-

\footnotetext{
Received July 19, 2011; revised Oct. 11, 2011; accepted 0ct. 12, 2011.

Author contributions: R.L. and D.J.S. designed research; R.L. performed research; R.L. and R.B. analyzed data; R.L., R.B., and D.J.S. wrote the paper.

This work was supported by the Medical Research Council (United Kingdom) and Research Councils United Kingdom.

This article is freely available online through the J Neurosci Open Choice option.

Correspondence should be addressed to Dr. Robert Leech, $\mathrm{C}^{3} \mathrm{NL}$, 3rd Floor Burlington Danes, Hammersmith Hospital, Du Cane Road, London W12 OHS, United Kingdom. E-mail: r.leech@imperial.ac.uk.

DOI:10.1523/JNEUROSCI.3689-11.2012

Copyright $\odot 2012$ the authors $\quad 0270-6474 / 12 / 320215-08 \$ 15.00 / 0$
}

trophysiological work with nonhuman primates suggests that parts of the PCC signal environmental change and the need to alter behavior (Hayden et al., 2009). This leads to the hypothesis that the PCC is involved in broad information gathering, which is necessary to control responses to a rapidly changing environment (Pearson et al., 2011; Leech et al., 2011).

The ability of an organism to flexibly react to change requires the integration of information from functionally distinct brain networks. If the PCC acts as a cortical hub integrating information across different functional networks, its brain activity should show a complex and dynamic pattern that partially reflects activity in other networks. We assumed that the pattern of neural activation within the PCC would be highly complex and separable into multiple signals that correlate with the brain activity observed in other networks. To investigate this, we used a novel data-driven multivariate analysis approach to parcellate the PCC into independent subregions and investigate their unique functional connectivity. Furthermore, although the whole of the PCC reliably deactivates on active tasks, we investigated whether distinct subregions within the PCC are modulated differentially by behavior on a simple choice reaction time (CRT) task (Bonnelle et al., 2011) and, in doing so, tested whether subregions of the PCC central to DMN deactivation could be isolated.

\section{Materials and Methods \\ Subjects \\ A group of 16 neurologically healthy subjects took part in the study (7 male; age range, $27-58$ years; mean age, 35.0 years). One subject had only resting-state fMRI data. The study was approved by the Hammersmith and Queen Charlotte's and Chelsea Research ethics committee.}




\section{Data-defined regions of interest}

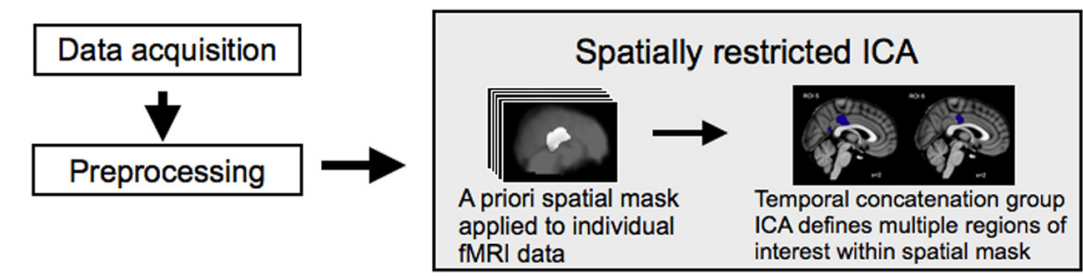

\section{Finding unique functional connectivity of ROls (rest or task)}

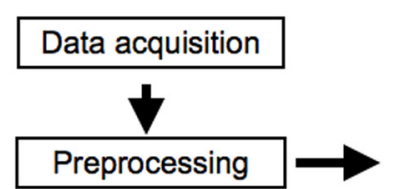

Preprocessing

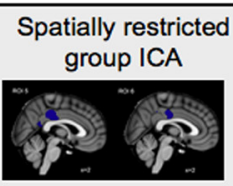

Regions of interest

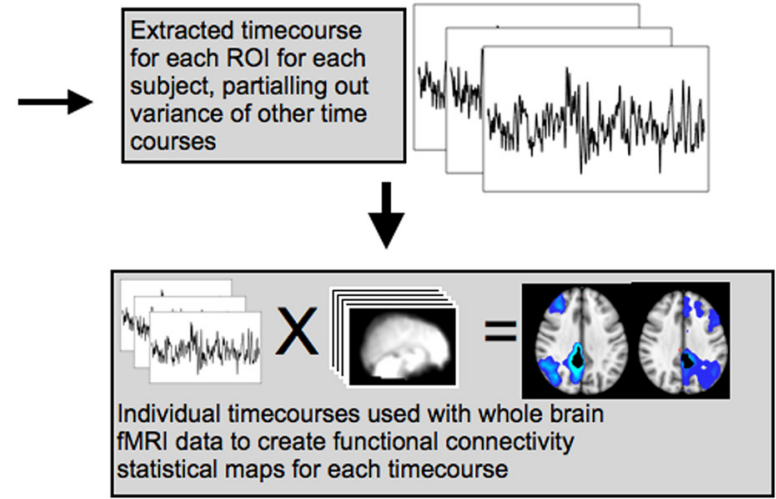

Figure 1. High-level schematic of the functional connectivity analysis. First, a group temporal concatenation ICA is performed to find separable spatial components within the PCC (top). These are then back projected to find a subject-specific time course for each PCC component. These time courses are then simultaneously entered into a GLM voxelwise throughout the brain, resulting in a functional connectivity map for each component.

\section{MRI image acquisition}

MRI data were obtained using a Philips Intera 3.0 tesla MRI scanner using Nova Dual gradients, a phased array head coil, and sensitivity encoding (SENSE) with an undersampling factor of 2. Functional MRI images were obtained using a $\mathrm{T} 2{ }^{\star}$-weighted gradient-echo echoplanar imaging (EPI) sequence with whole-brain coverage (TR, $2000 \mathrm{~ms}$; TE, 30 $\mathrm{ms}$; 31 ascending slices with thickness $3.25 \mathrm{~mm}$; gap $0.75 \mathrm{~mm}$; voxel size, $2.19 \times 2.19 \times 4 \mathrm{~mm}$; flip angle, $90^{\circ}$; field of view, $280 \times 220 \times 123 \mathrm{~mm}$; matrix, $112 \times 87)$. Quadratic shim gradients were used to correct for magnetic field inhomogeneities within the brain. T1-weighted wholebrain structural images were also obtained in all subjects. Paradigms were programmed using Matlab Psychophysics toolbox (Psychtoolbox-3; www.psychtoolbox.org) and stimuli presented through an IFIS-SA system (Invivo). Responses were recorded through a fiber optic response box (Nordicneurolab), interfaced with the stimulus presentation PC running Matlab.

In the task, subjects were presented with an initial fixation cross for 350 $\mathrm{ms}$. This was followed by a response cue in the form of an arrow $(<<$ or $\gg$ ) in the direction of the required response and lasting $1400 \mathrm{~ms}$. The interstimulus interval was $1750 \mathrm{~ms}$. Finger-press responses were made with the index finger of each hand. Subjects were instructed to respond as quickly and as accurately as possible. To maximize design efficiency stimulus, presentation was blocked, with five repeated blocks of 14 response trials, 14 rest trials, and four response trials at the start of the experiment, resulting in 74 response trials in total.

\section{Regional brain activation analysis of the CRT}

The first four volumes acquired were discarded to allow for T1 equilibrium effects. Image preprocessing involved realignment of EPI images to remove the effects of motion between scans, spatial smoothing using a 5 $\mathrm{mm}$ full-width half-maximum Gaussian kernel, prewhitening using FILM and temporal high-pass filtering using a cutoff frequency of $1 / 50$ $\mathrm{Hz}$ to correct for baseline drifts in the signal. FMRIB's Linear Image Registration Tool (FLIRT) (Smith et al., 2004) was used to register EPI functional datasets into standard MNI space using the participant's individual high-resolution anatomical images.

fMRI data were analyzed using voxelwise time series analysis within the framework of the general linear model (GLM). To this end, a design matrix was generated with a synthetic hemodynamic response function and its first temporal derivative. Task blocks were modeled in the design matrix with rest periods serving as the implicit baseline. In addition, six motion parameters were included in the model to control for signal associated with head movement. Mixed-effects analysis of session and group effects was performed using FLAME (FMRIB's Local Analysis of Mixed Effects) (Beckmann et al., 2003). Final statistical images were thresholded using Gaussian random field-based cluster inference with a height threshold of $Z>2.3$ and a cluster significance threshold of $p<$ 0.05 . This resulted in statistical maps of voxels significantly activated by the CRT and a separate map of voxels showing a relative deactivation on CRT.

\section{Functional connectivity analysis}

Data preprocessing. As in the whole-brain activation analysis, the functional connectivity analysis (Fig. 1) approach starts by preprocessing the data; i.e., motion correction, spatial smoothing, and temporal filtering. In addition, variance associated with motion ( 6 variables) and the time courses of white matter and CSF were removed from the whole-brain functional data using ordinary least-squares linear regression. Mean time courses were extracted from a 3-mm-radius sphere within the white matter (MNI: $-26,-22,28$ ) and from one lateral ventricle (MNI: 2, $10,8)$. Average whole-brain or gray matter activity was not regressed, as this can complicate the interpretation of negative functional correlation findings.

Finding subregions within the PCC. We performed our analysis on a PCC region defined using combined structural and functional criteria. First, voxels anatomically part of the PCC were selected, defined using the Harvard-Oxford probabilistic anatomical atlas within fslview $(>20 \%$ probability). In addition, to ensure that the selected voxels were func- 

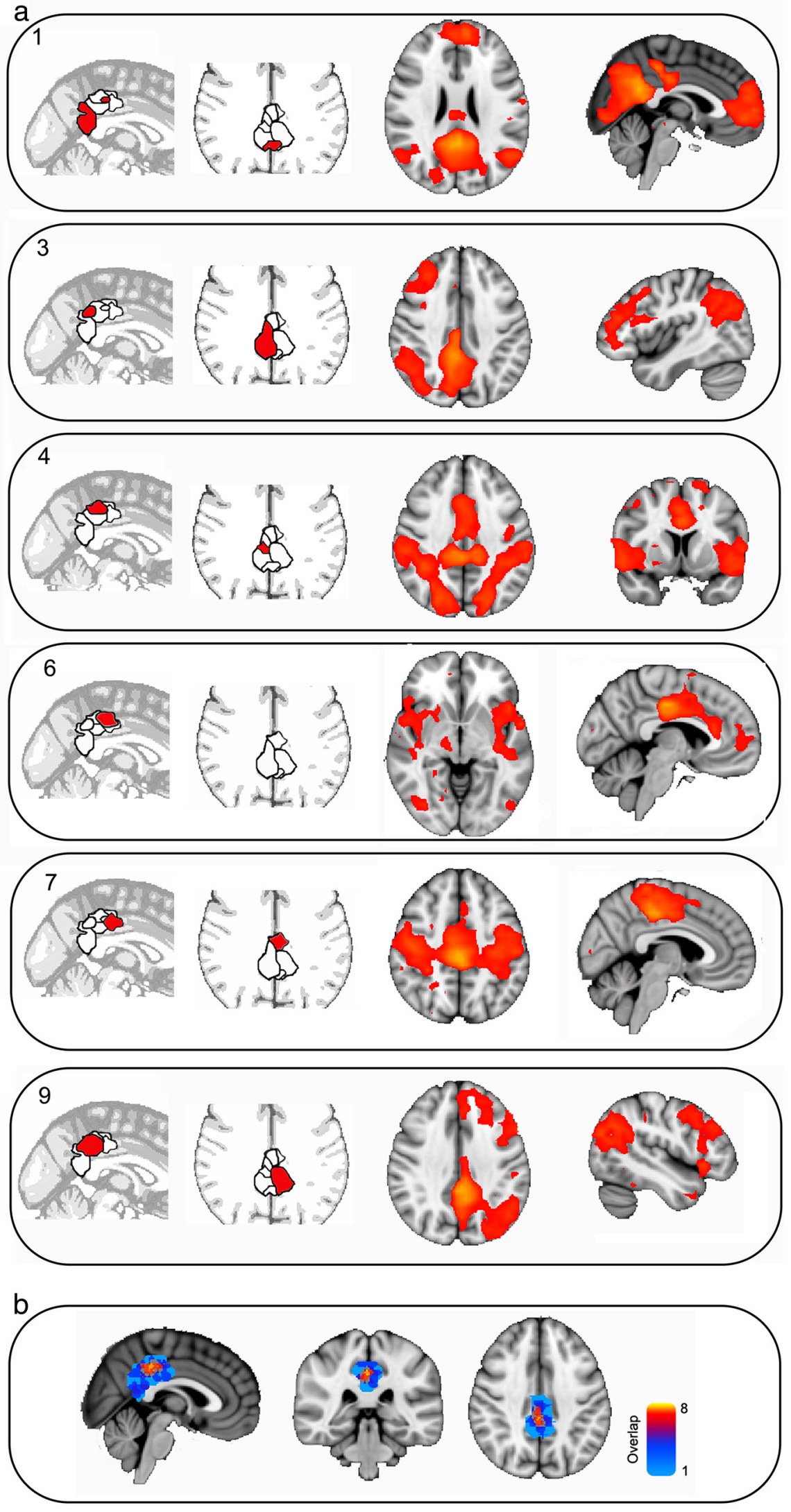

Figure 2. a, The locations (left) and the resulting maps of functional connectivity (right) of six or the 10 data-derived regions of interest. The results of the connectivity analysis are thresholded at $p<0.05$, corrected for multiple comparisons. $\boldsymbol{b}$, The overlap between the regions of interest, demonstrating the highest overlap in dorsal PCC. Warm colors indicate higher overlap. tionally within the DMN, the PCC anatomical mask was inclusively masked by a map of voxels that significantly deactivated during CRT performance. A temporal concatenation group independent component analysis (ICA) (Beckmann et al., 2005) was then run on the resting fMRI data within this PCC/DMN mask. This approach identified subregions within the PCC with spatially and temporally distinct patterns of neural activity. The analysis was initially constrained to extract 10 independent components. The dimensionality of the ICA reflects a trade-off between granularity and noise. To confirm that our results hold for other ICA dimensionalities, the analysis was also run with seven and 15 components, with qualitatively similar results.

Finding whole-brain functional connectivity with PCC subregions. For the next step, a GLM was used with the fMRI data simultaneously including the 10 spatial maps from the ICA as a design matrix; this approach results in a subject-specific time course for each spatial map controlling for the variance explained by the other spatial maps. It can be thought of as finding 10 independent signals (corresponding primarily to different but overlapping regions of interest) from within the PCC for each subject. We then investigated whether these independent signals correlated with activity in the rest of the brain. A second GLM was used, this time entering whole-brain $\mathrm{fMRI}$ data. The time courses of the PCC subregions calculated in the previous step were simultaneously included in the design matrix to generate a set of whole-brain statistical maps [this approach is a variant on the dual regression approach (Zuo et al., 2010), used by Leech et al. (2011), Bonnelle et al. (2011), and Sharp et al. (2011)]. The resulting statistical maps provide a whole-brain voxelwise measure of functional connectivity with each of the signals controlling for the other signals.

Finally, the resulting spatial maps were combined and a higher-level general linear model (Beckmann et al., 2003) was computed and statistically evaluated using both parametric and nonparametric statistics (random permutation testing). Parametric and nonparametric statistics provided highly consistent results, so only parametric statistics are reported in the Results, below. Family-wise error corrections were applied to statistics to avoid overrejection of the null hypothesis because of multiple comparisons. To help interpret the findings, it is important to be able to label the resulting networks in a principled way with reference to existing resting state networks (RSN). To do this, the spatial maps corresponding to each ROI were correlated with the results from running a group temporal concatenation ICA on the whole-brain data with 20 components. The highest resulting correlation with different RSNs from the ICA was used to label the ROIderived spatial maps. As a further validation step, the consistency of this labeling was also assessed by correlating the ROI-derived functional connectivity maps with the RSNs identified (and labeled) in Beckmann et al. (2005). The labeling of the RSNs identified in Beckmann et al. (2005) (e.g., the labeling of the right fronto- 
parietal network as related to attentional control) have been validated with regard to a large database of task data (Smith et al., 2009). We have, therefore, used these labels to interpret the results of our analyses. The results of the two approaches to labeling the ROI-derived networks are qualitatively similar. To illustrate the advantages of our multivariate approach over the more traditional univariate approach, a functional connectivity analysis was undertaken, but without using multiple regression.

Evaluating task modulation in different subregions. Initially, functional connectivity was calculated for the resting state dataset. Subsequently, functional connectivity for the same 10 regions of interest delineated at rest was also evaluated for the CRT dataset. Therefore, we could evaluate whether the functional connectivity of the subsignals of the PCC is modulated by task. Equally importantly, the CRT (events convolved with a canonical hemodynamic response function) provides an expected time course to be entered into a GLM with the time courses of each region of interest to investigate whether the signal from individual ROIs is modulated by the task. Given that the PCC region of interest was selected based on deactivation in the CRT task, the initial expectation was that all 10 subsignals of the PCC should show a deactivation on the same CRT task. It is therefore noteworthy if only a subset of the signals are selectively deactivated using the multivariate analysis with other subsignals showing no task modulation.

\section{Results}

Functionally distinct but spatially overlapping subregions exist within posterior cingulate cortex

When attention is focused externally, rapid and reactive deactivation is usually observed across the PCC (Singh and Fawcett, 2008). However, a more complex organization is suggested by recent studies of the region's structural and functional connectivity (Hagmann et al., 2008; Margulies et al., 2009; Leech et al., 2011). Here, we extend this work by using a novel extension of the multivariate ICA approach that allows distinct but overlapping signals to be separated in a data-driven way from resting-state fMRI. Within the PCC, 10 partially overlapping subregions were identified, each of which had a distinct time course of BOLD fluctuation (four of these regions and the overlap of all regions are illustrated in Fig. $2 a$ ). The analysis was performed on voxels that were both anatomically part of the PCC, and functionally part of the DMN (see Materials and Methods, above). Hence, these initial results were uninfluenced by patterns of activity in the rest of the brain. The approach allows complex signals that overlap both spatially and temporally to be separated, providing a more accurate description of the region's functional connectivity.

Subregions within the PCC identified by ICA are functionally connected to distinct distributed brain networks

Next, we investigated whether these separate PCC signals correlated with activity in other brain networks (Fig. 2). Our PCC
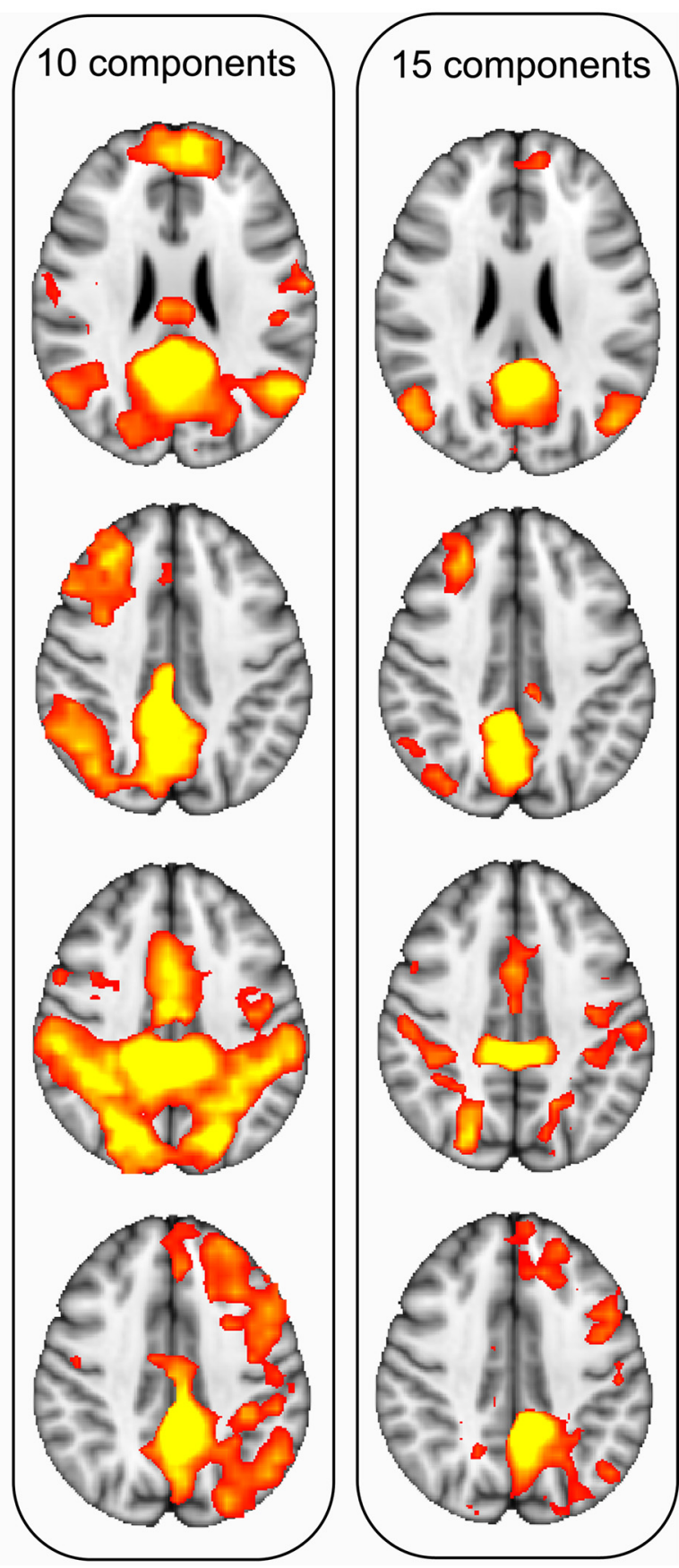

Figure 3. Consistency of resulting networks with seven (left), 10 (middle), and 15 (right) components (different columns).

search area was constrained to be functionally part of the DMN, and would a priori be expected to have highly correlated activity with the rest of the DMN (Fransson and Marrelec, 2008). This dominant pattern was observed in a predominantly ventral part of the PCC (Fig. 2a, ROI 1). However, it masks more subtle functional interactions of the PCC with other brain regions. Five subregions within the dorsal PCC shared a well organized pattern of functional connectivity with networks not typically associated with the DMN. These were left- and right-lateralized frontoparietal networks covering inferior parietal and distributed frontal regions, a motor network, a sensory network, and a more executive network (six of these networks are presented in Fig. 2a). These established networks were located in a data-driven manner by correlating the time series from covaried PCC subregions with 


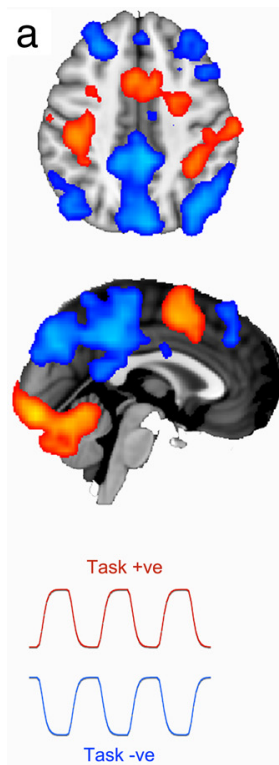

b
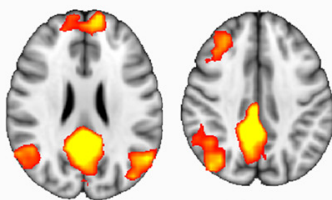
ROI 3
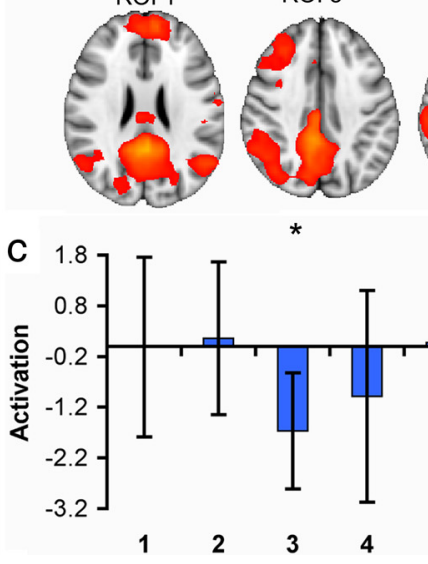

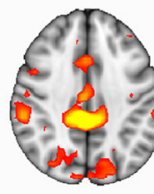
ROI 4
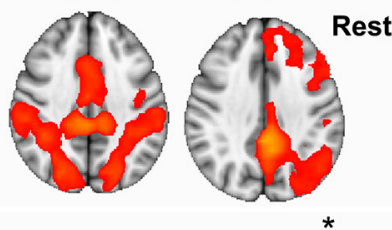

*

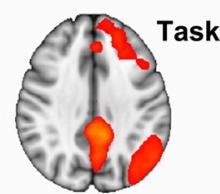

Rest

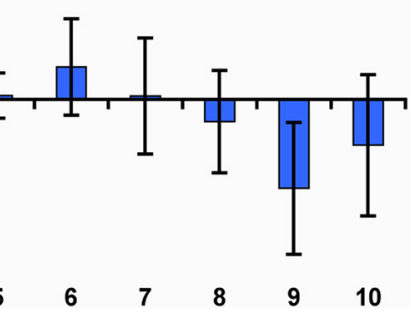

Figure 4. The effect of a simple attentional task on functional connectivity within the PCC. $\boldsymbol{a}$, Regions positively activated (warm colors) or deactivated (in cool colors) by a choice reaction time task compared with rest $(p<0.05$, cluster corrected for multiple comparisons). The time courses in red and blue illustrate the task-positive and task-negative phases. Representative time courses are shown for task and fixation. $\boldsymbol{b}$, A comparison of functional connectivity maps for four regions of interest during task (i.e., blocks of active task and fixation) and during a separate rest scan with no explicit task. The spatial correlation between the functional connectivity during the resting scan and during the task scan are as follows: ROI $1, r=0.86 ; \mathrm{ROI} 3, r=0.77 ; \mathrm{RO}$ I $4, r=$ $0.93 ; \mathrm{ROI} 9, r=0.55$. C, Task-activation of regions of interest. Asterisks indicate regions that are significantly modulated (in this case, deactivated) on task.

the activity of the rest of the brain. Several subregions shared low spatial similarity with any well characterized brain networks, and possibly reflect nonbrain signal, for example, physiological noise.

Although the exact composition of the regions of interest is dependent on the number of components extracted, qualitatively similar results are obtained when separating the PCC into seven, 10, or 15 components (Fig. 3). The topology of the spatial maps found with ICA is sensitive to the number of components derived. To demonstrate that the results are representative of the underlying data and not an artifact of the specific number of independent components chosen, the analysis was repeated with seven and 15 independent components. The resulting wholebrain spatial functional connectivity maps from applying a seven- and 15-component analysis to the PCC were calculated, in addition to the 10-component case. The 10-component functional connectivity spatial maps were used as a template; the spatial similarity (correlation) between each of these maps and the maps derived from seven and 15 components were calculated. For each spatial map identified in the 10-component case (e.g., $\mathrm{DMN}$, frontoparietal and executive networks), the corresponding map in the seven- and 15-component cases was labeled based on the map with the highest spatial correlation. For the seven-component case, the spatial correlations with the 10component case were as follows: ROI1, $r=0.93$; ROI3, $r=0.79$; ROI4, $r=0.94$; ROI9, $r=0.85$. For the 15 -component case, the correlations were as follows: ROI1, $r=0.48$; ROI3, $r=0.70$; ROI4, $r=0.95$; ROI9, $r=0.87$.

Subregions within the posterior cingulate cortex show distinct changes in neural activity during an attentionally demanding task

We next investigated the function of these distinct PCC subregions by studying their response during a simple yet attentionally demanding visual forced CRT task (Fig. 4). A blocked design was used, so periods of CRT were interleaved with short periods of fixation (Fig. 4a). Spatial patterns of functional connectivity were highly stable during this type of task performance, with spatial correlation analysis showing a large overlap in the functional connectivity between rest scans with no explicit task and the CRT task scans. Previous work has shown general deactivation of the PCC in this type of task (Bonnelle et al., 2011; Sharp et al., 2011) and the PCC in this study was defined in part by its deactivation on task. However, once overall activity within the PCC was controlled for, only the dorsal PCC subregions connected to left and right frontoparietal networks showed relative modulation during task performance (left: $t_{(14)}=-2.90, p<$ 0.0059 ; right: $t_{(14)}=-3.12, p<0.0038$; Fig. $4 c$ ). This demonstrates that in the context of this simple but attentionally demanding task, the expected pattern of task-induced deactivation is isolated to two parts of the dorsal PCC, which are functionally connected to frontoparietal attentional networks. No subregions had time courses that positively correlated with task (i.e., that increased in activation during task positive blocks). In contrast, in the univariate case, all 10 regions of interest deactivated on task relative to rest.

\section{Comparison of the use of multiple regression with univariate functional connectivity analysis}

Direct comparison of univariate and multivariate approaches demonstrates the advantage of controlling for overall patterns of functional connectivity to reveal differential contributions of different subregions of the PCC. Using multiple regression to calculate the time courses of each ROI ensures that the time courses capture more of what is unique to that cortical subregion. To illustrate the advantage of covarying out adjacent time courses, the multivariate statistics are compared with a more traditional analysis approach to functional connectivity. For this approach, the ICA-derived spatial maps were thresholded with a $z>2$ and binarized to create 10 distinct regions of interest. The mean time course was then calculated for each ROI (univariate analysis). Figure $5 a$ contains results from both the univariate and multivariate analyses. The figure presents the similarities in the time courses between ROIs. The similarity matrices were calculated for each participant. The correlation coefficients were converted to $z$-values using the Fisher transform and then averaged across subjects. The average $z$-value of pairwise similarity was then inverse transformed, resulting in an average correlation coefficient. For the univariate analysis, the time courses across the ROIs are highly similar (mean $r, 0.7402$; SD, 0.3368; range, $0.4088-$ 0.9183 ). However, with the multivariate approach, the time courses become considerably more distinct (mean $r, 0.2478$; SD, 0.3555 ; range, $-0.0689-0.5688)$.

We also investigated the effects of taking the multivariate approach on the whole-brain maps of functional connectivity. The univariate approach used the time course extracted from each ROI in a separate GLM. The resulting statistical maps reveal 
strong functional connectivity between each ROI and a large extent of cortical and subcortical brain regions that overlaps considerably across ROIs. In contrast, our multivariate approach results in more discrete brain regions that more closely approximate well described functional brain networks. A selection of the resulting networks are presented in Figure $5 b$.

Finally, investigating the effect of task modulation (the choice reaction task) on the time courses reveals a very different pattern for the multivariate rather than the univariate approach (Fig. $5 c$ ). For the univariate approach, all time courses show significant deactivation with the executive task. This result is unsurprising given that the ROIs are all constrained to be within a part of the DMN that deactivates on this task. However, the multivariate approach results in only ROIs 3 and 9 (the lateralized frontoparietal networks) being significantly modulated by task. Again, this emphasizes the advantage of taking a multivariate approach, resulting in a far more specific interpretation of which ROIs are involved in the task-based deactivation within the PCC.

\section{Discussion}

We show that neural activity within the PCC has a highly complex structure. Other work using graph-theoretic structural and functional measures suggests that the PCC acts as a connector hub, a highly connected region for cortical and subcortical networks (Hagmann et al., 2008); our results provide direct evidence that this is the case. We separated neural activity within the PCC into functionally distinct but spatially overlapping subregions using multivariate analysis, and show that signals from these areas correlate with activity in a number of well described largescale brain networks. This demonstrates that this relatively small region has a complex functional organization. Further, we show that the relative deactivation of the PCC found with performance of an attentionally demanding task is isolated to signals originating in dorsal PCC. These signals echo activity in frontoparietal networks that, given their spatial locations and previous studies (Corbetta and Shultz, 2002; Beckmann et al., 2005; Smith et al., 2009), are likely involved in attentional control. This suggests that the dorsal PCC has greater communication with attentional systems during periods without a focused task, possibly reflecting the PCC's role in maintaining a broad attentional focus.

Previous work has demonstrated that the medial posterior parietal regions (the PCC and precuneus) are highly functionally heterogeneous in humans and nonhuman primates (Vogt et al., 2006; Margulies et al., 2009), with functionally separable dorsal and ventral subregions (Dastjerdi et al., 2011; Leech et al., 2011). Our current work suggests that the functional complexity within the PCC is even greater than previously described, with partially overlapping and closely adjacent subregions displaying different patterns of functional connectivity. More importantly, we demonstrate that the different neural signals isolated within the PCC may be functionally discrete, reflecting independent contributions from different whole-brain neural networks. Subregions within the PCC interact with putative executive, attentional, motor, language, and default mode networks in close proximity within the PCC.

In nonhuman primates, single-cell recordings from the PCC suggest that the region plays a more active role in cognition than previously suggested (Hayden et al., 2009; Pearson et al., 2011). Cells code the consequences of choices from moment-tomoment and predict the likelihood of a strategic change in behavior (Hayden et al., 2008; Pearson et al., 2009). In addition, on simple tests of decision-making, response speed is predicted by spontaneous fluctuations in neural activity within the PCC before the response (Hayden et al., 2009). This has led to the proposal that the PCC signals environmental change and the need to alter behavior (Pearson et al., 2011), which is in keeping with converging neuroimaging work in humans suggesting a role for this region in cognitive control (Shulman et al., 1997; Hampson et al., 2006; Gilbert et al., 2007; Kelly et al., 2008; Bonnelle et al., 2011; Leech et al., 2011). The activity of functionally distinct distributed brain networks is echoed in spatially overlapping but distinct parts of the PCC. Whether these subsignals are repetitions of the neural signals found in distant networks (i.e., true echoes of activation found elsewhere in the brain) or indicate subregions of the PCC that are part of these large-scale brain networks is unclear and will require further investigation. Either way, this result suggests a potential role of the PCC for integrating 
diverse types of information needed to monitor for environmental change.

Our results are not well described by a simple model of the $\mathrm{DMN}$ as an isolated network engaged during internally directed thought and switched off when attention needs to be directed externally (Buckner et al., 2008). Surprisingly, once the activity of the PCC was isolated into multiple signals, activation in the subregion showing most functional connection to the DMN was unaffected by CRT performance. Instead, relative deactivation is specifically seen in the dorsal PCC subregions that are functionally connected to lateralized frontoparietal networks. The lateralized frontoparietal systems, traditionally reported during cognitively demanding tasks, are here seen connected to the PCC during task-negative periods, making the echo metaphor more apt; the traditional frontoparietal temporal modulation of the signal (up on task) is inverted in the putative PCC reflection. These frontoparietal networks may be involved in attentional control (Corbetta et al., 2002) and this activity change within the PCC might reflect varying levels of information gathering in different phases of task performance. If the PCC is playing a role in environmental change detection, interactions with attentional networks might be expected to be high during periods of fixation, when cues for action are awaited and broad, unfocused information gathering is required. Once focused task performance has started, this type of interaction becomes less relevant, so PCC activity falls in preference for the specific attentional demands of the task during task performance.

The highly heterogeneous nature of the PCC's functional connectivity is not compatible with a unitary cognitive function. Despite our PCC search area being partly defined by the presence of task deactivation, once overall signal changes had been controlled for, only two subregions displayed the relative deactivation during task performance typically associated with the DMN. This contrasts with many previous studies of DMN function and suggests that much of the PCC is continuously active during the type of attentionally demanding task we have investigated. The high metabolic rate frequently observed within the PCC (Raichle et al., 2001) is consistent with this possible continuous activity and may in part explain the persistent high metabolic rate and the elevated levels of blood flow observed within the PCC relative to other cortical brain regions during executive tasks, as well as rest (Pfefferbaum et al., 2011).

The results suggest a role for the PCC as a central hub for information exchange in the brain. However, the current research has a number of limitations that could be addressed in future work. To better understand PCC functionality, it is necessary to investigate how other task demands (e.g., internally directed attention such as autobiographical memory) modulate the other signals observed within the PCC, as this may reveal further functional specialization. It would also be important to replicate the current results with a larger sample size, where increased power may allow us to investigate whether the PCC can be further fractionated into even more subregions. It would be equally useful to investigate different brain regions outside of the ventral medial parietal lobe that may also be decomposable into multiple subsignals. Previous structural and functional imaging analyses suggest that there are other hub regions outside the PCC, including medial structures such as the thalamus or the anterior cingulate. For example, the anterior cingulate has been suggested to play a pivotal role in multiple discrete distributed networks (Paus, 2001). Indeed, one possible hypothesis for future work is that a large area of cortex taken from any region of the brain could be similarly decomposed into subsignals, possibly reflecting the integration of information of large-scale distributed networks.

\section{References}

Beckmann CF, Jenkinson M, Smith SM (2003) General multilevel linear modeling for group analysis in FMRI. Neuroimage 20:1052-1063.

Beckmann CF, DeLuca M, Devlin JT, Smith SM (2005) Investigations into resting-state connectivity using independent component analysis. Philos Trans R Soc Lond B Biol Sci 360:1001-1013.

Bonnelle V, Leech R, Kinnunen KM, Ham TE, Beckmann CF, De Boissezon X, Greenwood RJ, Sharp DJ (2011) Default mode network connectivity predicts sustained attention deficits following traumatic brain injury. J Neurosci 31:13442-13451.

Buckner RL, Andrews-Hanna JR, Schacter DL (2008) The brain's default network: anatomy, function, and relevance to disease. Ann N Y Acad Sci 1124:1-38.

Corbetta M, Shulman GL (2002) Control of goal-directed and stimulusdriven attention in the brain. Nat Rev Neurosci 3:201-215.

Dastjerdi M, Foster BL, Nasrullah S, Rauschecker AM, Dougherty RF, Townsend JD, Chang C, Greicius MD, Menon V, Kennedy DP, Parvizi J (2011) Differential electrophysiological response during rest, selfreferential, and non-self-referential tasks in human posteromedial cortex. Proc Natl Acad Sci U S A 108:3023-3028.

Fransson P, Marrelec G (2008) The precuneus/posterior cingulate cortex plays a pivotal role in the default mode network: evidence from a partial correlation network analysis. Neuroimage 42:1178-1184.

Gilbert SJ, Dumontheil I, Simons JS, Frith CD, Burgess PW (2007) Comment on "Wandering minds: the default network and stimulusindependent thought." Science 317:43; author reply 43.

Hagmann P, Cammoun L, Gigandet X, Meuli R, Honey CJ, Wedeen VJ, Sporns O (2008) Mapping the structural core of human cerebral cortex. PLoS Biol 6:e159.

Hampson M, Driesen NR, Skudlarski P, Gore JC, Constable RT (2006) Brain connectivity related to working memory performance. J Neurosci 26:13338-13343.

Hayden BY, Nair AC, McCoy AN, Platt ML (2008) Posterior cingulate cortex mediates outcome-contingent allocation of behavior. Neuron 60:19-25.

Hayden BY, Smith DV, Platt ML (2009) Electrophysiological correlates of default-mode processing in macaque posterior cingulate cortex. Proc Natl Acad Sci U S A 106:5948-5953.

Kelly AM, Uddin LQ, Biswal BB, Castellanos FX, Milham MP (2008) Competition between functional brain networks mediates behavioral variability. Neuroimage 39:527-537.

Leech R, Kamourieh S, Beckmann CF, Sharp DJ (2011) Fractionating the default mode network: distinct contributions of the ventral and dorsal posterior cingulate cortex to cognitive control. J Neurosci 31:3217-3224.

Margulies DS, Vincent JL, Kelly C, Lohmann G, Uddin LQ, Biswal BB, Villringer A, Castellanos FX, Milham MP, Petrides M (2009) Precuneus shares intrinsic functional architecture in humans and monkeys. Proc Natl Acad Sci U S A 106:20069-20074.

Marrelec G, Kim J, Doyon J, Horwitz B (2009) Large scale neural model validation of partial correlation analysis for effective connectivity investigation in functional MRI. Hum Brain Mapp 30:941-950.

Paus T (2001) Primate anterior cingulate cortex: where motor control, drive and cognition interface. Nat Rev Neurosci 2:417-424.

Pearson JM, Hayden BY, Raghavachari S, Platt ML (2009) Neurons in posterior cingulate cortex signal exploratory decisions in a dynamic multi option choice task. Curr Biol 19:1532-1537.

Pearson JM, Heilbronner SR, Barack DL, Hayden BY, Platt ML (2011) Posterior cingulate cortex: adapting behavior to a changing world. Trends Cogn Sci 15:143-151.

Pfefferbaum A, Chanraud S, Pitel AL, Müller-Oehring E, Shankaranarayanan A, Alsop DC, Rohlfing T, Sullivan EV (2011) Cerebral blood flow in posterior cortical nodes of the default mode network decreases with task engagement but remains higher than in most brain regions. Cereb Cortex 21:233-244

Raichle ME, MacLeod AM, Snyder AZ, Powers WJ, Gusnard DA, Shulman GL (2001) A default mode of brain function. Proc Natl Acad Sci U S A 98:676-682. 
Sharp DJ, Beckmann CF, Greenwood R, Kinnunen KM, Bonnelle V, De Boissezon X, Powell JH, Counsell SJ, Patel MC, Leech R (2011) Default mode network functional and structural connectivity after traumatic brain injury. Brain 134:2233-2247.

Shulman GL, Fiez JA, Corbetta M, Buckner RL, Miezin FM, Raichle ME, Petersen SE (1997) Common blood flow changes across visual tasks. II. Decreases in cerebral cortex. J Cogn Neurosci 9:648-663.

Singh KD, Fawcett IP (2008) Transient and linearly graded deactivation of the human default-mode network by a visual detection task. Neuroimage $41: 100-112$.

Smith SM, Jenkinson M, Woolrich MW, Beckmann CF, Behrens TE, Johansen-Berg H, Bannister PR, De Luca M, Drobnjak I, Flitney DE, Niazy RK, Saunders J, Vickers J, Zhang Y, De Stefano N, Brady JM, Matthews PM (2004) Advances in functional and structural MR im- age analysis and implementation as FSL. Neuroimage 23 [Suppl 1]:S208-S219.

Smith SM, Fox PT, Miller KL, Glahn DC, Fox PM, Mackay CE, Filippini N, Watkins KE, Toro R, Laird AR, Beckmann CF (2009) Correspondence of the brain's functional architecture during activation and rest. Proc Natl Acad Sci U S A 106:13040-13045.

Smith SM, Miller KL, Salimi-Khorshidi G, Webster M, Beckmann CF, Nichols TE, Ramsey JD, Woolrich MW (2011) Network modelling methods for FMRI. Neuroimage 54:875-891.

Vogt BA, Vogt L, Laureys S (2006) Cytology and functionally correlated circuits of human posterior cingulate areas. Neuroimage 29:452-466.

Zuo XN, Kelly C, Adelstein JS, Klein DF, Castellanos FX, Milham MP (2010) Reliable intrinsic connectivity networks: test-retest evaluation using ICA and dual regression approach. Neuroimage 49:2163-2177. 